\title{
Les projets de sciences citoyennes à l'école : pour quelles visées éducatives ? L'exemple du projet «Les gardiens des chênes »
}

Séverine Perron, Patricia Marzin-Janvier et Bastien Castagneyrol

\section{(2) OpenEdition}

\section{Journals}

Édition électronique

URL : https://journals.openedition.org/ere/7977

DOI : $10.4000 /$ ere. 7977

ISSN : 2561-2271

Éditeur

Centr'ERE

\section{Référence électronique}

Séverine Perron, Patricia Marzin-Janvier et Bastien Castagneyrol, «Les projets de sciences citoyennes à l'école : pour quelles visées éducatives ? L'exemple du projet « Les gardiens des chênes » », Éducation relative à l'environnement [En ligne], Volume 16-2 | 2021, mis en ligne le 14 octobre 2021, consulté le 08 février 2022. URL : http://journals.openedition.org/ere/7977 ; DOl : https://doi.org/10.4000/ere.7977

Ce document a été généré automatiquement le 8 février 2022.

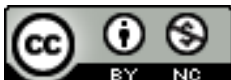

La revue Éducation relative à l'environnement est mise à disposition selon les termes de la Licence Creative Commons Attribution - Pas d'Utilisation Commerciale 4.0 International. 


\title{
Les projets de sciences citoyennes à l'école : pour quelles visées éducatives? L'exemple du projet «Les gardiens des chênes »
}

\author{
Séverine Perron, Patricia Marzin-Janvier et Bastien Castagneyrol
}

1 Ces dernières années, l'enseignement scientifique et technologique a connu une profonde reconfiguration dans plusieurs pays occidentaux, dont la France. En effet, certains textes institutionnels et scientifiques indiquent que l'éducation scientifique et technologique consiste en la formation d'une relève scientifique et technique, et qu'elle correspond aussi au développement d'une culture scientifique et technologique pour tous (Roberts, 2007 ; DeBoer, 2000). D'autres textes évoquent l'importance de susciter l'intérêt des élèves envers les sciences notamment en contextualisant les apprentissages à travers la mise en œuvre de démarches d'investigation scientifique (DIS) et l'étude des enjeux sociétaux associés aux sciences (Eurydice, 2006; Hasni et Potvin, 2015). Par ailleurs, dans une société où il semble indispensable de renforcer les liens entre les sciences et la cité, des projets de sciences citoyennes à destination des élèves, se développent (Castagneyrol et coll., 2019; Bosdeveix et coll., 2018). Ces derniers semblent prometteurs quant à leurs potentialités d'apprentissages par les élèves (Leuenberger, W., Larsen, Leuenberger, J., et Parry, 2019). Dans ce contexte, il apparait nécessaire de questionner ces projets au regard notamment de leurs finalités (pour les scientifiques et pour les enseignants), de leurs mises en œuvre et des apprentissages réalisés par les élèves. C'est pourquoi, la présente étude a pour objectif d'identifier principalement les visées éducatives des enseignants lorsqu'ils font participer leurs élèves au projet «Les gardiens des chênes » développé et déployé par des chercheurs de l'INRAE (l'Institut national de recherche pour l'agriculture, l'alimentation et l'environnement, France).

2 Après avoir évoqué le contexte actuel de l'enseignement-apprentissage des sciences et présenté les «sciences participatives», nous explicitons le cadre conceptuel, la 
méthode de recherche ainsi que les résultats de notre étude. Des perspectives de recherches sont exposées au sein de la conclusion.

\section{L'enseignement des sciences aujourd'hui}

3 Dans cette section nous abordons les enjeux et les finalités éducatives de l'enseignement des sciences.

\section{Le double enjeu de l'enseignement des sciences}

Selon certains auteurs (Roberts, 2007; DeBoer; 2000), les enjeux de l'éducation scientifique et technologique font référence à deux aspects: (a) la formation d'une relève scientifique et technique et (b) le développement d'une culture scientifique et technologique pour tous.

5 Le premier aspect porte sur la nécessité pour les sociétés de former des personnes ayant des compétences en sciences et technologie ( $\mathrm{S}$ et $\mathrm{T}$ ) à la fois dans des filières scientifiques et technologiques, mais également dans plusieurs secteurs d'emploi. Dans cette vision, l'enseignement des $\mathrm{S}$ et $\mathrm{T}$ doit permettre aux élèves une meilleure acculturation à cette discipline afin d'acquérir un socle scientifique et technologique solide leur assurant de poursuivre des études en $\mathrm{S}$ et $\mathrm{T}$ ou d'avoir accès à des professions qui nécessitent une formation en $\mathrm{S}$ et $\mathrm{T}$. Dans cette perspective, un manque de personnes formées dans les domaines associés aux $\mathrm{S}$ et $\mathrm{T}$ priverait la société de ressources humaines indispensables à son développement industriel, mais aussi et surtout économique. En ce sens, l'Organisation de Coopération et de Développement Économiques (OCDE, 2007) soutient que, pour relever les défis scientifiques de notre époque, tels que le réchauffement climatique, l'épuisement des carburants fossiles, ou encore les traitements de pathologies comme le sida ou les cancers, les pays devront réaliser des investissements importants dans leurs infrastructures scientifiques et attirer ou développer une main-d'œuvre hautement qualifiée dans les domaines scientifiques et technologiques.

6 Le deuxième aspect concerne les enjeux de l'éducation des $S$ et $T$ en lien avec le développement d'une culture scientifique et technologique pour tous les élèves, qu'ils se destinent ou non à poursuivre une carrière dans les domaines associés aux $\mathrm{S}$ et $\mathrm{T}$. Cette autre approche de l'éducation des $\mathrm{S}$ et $\mathrm{T}$ envisage l'enseignement de ces disciplines comme une formation qui doit d'abord servir à tous les élèves, quelles que soient leurs ambitions scolaires, personnelles et professionnelles. DeBoer (2000) souligne que la culture scientifique concerne l'ensemble de la population, car elle touche l'intérêt pour la science et va au-delà des apprentissages prescrits à l'école : " The important thing is that students learn something that they will find interesting so that they will continue to study science both formally and informally in the future ${ }^{1}$ (p. 597). Dans cette perspective, les visées de l'enseignement des $\mathrm{S}$ et $\mathrm{T}$ se détachent de la simple initiation aux différentes disciplines scientifiques de référence et de la formation des futurs chercheurs ou ingénieurs. 


\section{Des finalités éducatives variées}

7 Hasni et Bousadra (2017) en réalisant une analyse systématique des publications qui se sont intéressées aux finalités des sciences à l'école, ont montré que malgré certaines différences les finalités privilégiées par les auteurs convergent autour de trois principales dimensions (figure 1): la dimension disciplinaire, la dimension épistémologique et la dimension sociale.

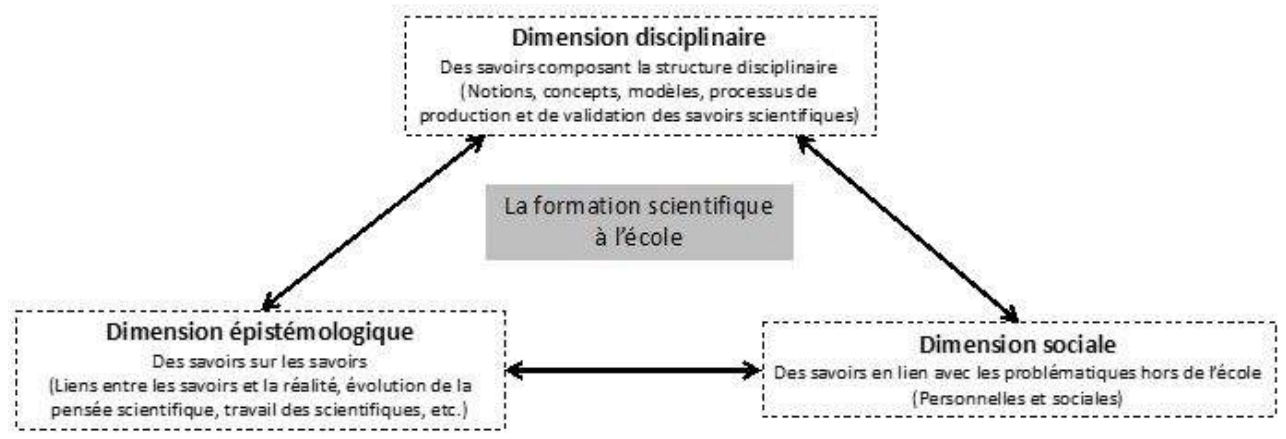

Figure 1 : Principales dimensions des finalités de l'éducation scientifique à l'école (Inspiré de Hasni et Bousadra ; 2017)

8 La dimension disciplinaire renvoie aux travaux de Schwab (1964) et a ce qu'il a nommé la structure des disciplines avec ses deux composantes, la substantive structure et la syntactical structure. La première composante comprend les savoirs conceptuels et la deuxième correspond aux savoirs et aux compétences méthodologiques en lien avec les démarches d'investigation scientifiques (DIS). Nous précisons que les deux composantes n'ont de sens que par leur articulation. En effet, les processus scientifiques ne peuvent être déployés que pour répondre à des questions et résoudre des problèmes ancrés dans des cadres théoriques existants et réciproquement.

9 La dimension épistémologique fait référence aux savoirs sur les savoirs scientifiques. Il s'agit d'amener les élèves à s'interroger sur la nature des savoirs scientifiques et sur les processus de leur production, sur la relation entre les savoirs et la réalité ainsi que sur l'évolution historique des savoirs et de la pensée scientifique.

10 La troisième dimension, la dimension sociale correspond à la formation des élèves leur permettant de transférer dans leur vie personnelle et sociale les savoirs en sciences et sur les sciences qu'ils ont construits. La prise en considération de cette dimension n'implique pas seulement d'aborder l'enseignement scientifique en lien avec la vie hors de l'école (la contextualisation) (Bennett, Lubben et Hogarth, 2007), mais également, et surtout, de permettre aux élèves de faire appel aux savoirs scientifiques pour prendre des décisions éclairées concernant leur vie personnelle (environnement, nutrition, sexualité, etc.) et pour exercer une citoyenneté éclairée face à des problématiques socio scientifiques (Albe, 2008; Kolstoe, 2000; Lee et Roth, 2003). Plusieurs de ces problématiques sont porteuses de ce que des auteurs qualifient de controverses socioscientifiques, parce qu'elles font l'objet d'un désaccord et d'un débat autant au niveau scientifique qu'au niveau social (Albe, 2008 ; Lederman, Antink et Bartos, 2014). Il s'agit par exemple de certaines problématiques environnementales comme le réchauffement climatique. La participation aux débats sur ces questions nécessite un éclairage scientifique, à côté d'autres éclairages sociaux, économiques, éthiques, etc. Outre les 
savoirs conceptuels et les processus scientifiques, ces problématiques font également appel aux valeurs (Hasni et Lebrun, 2008).

11 Récemment, en France et dans de nombreux autres systèmes éducatifs, les finalités et les contenus des enseignements scolaires ont été profondément renouvelés en faisant notamment appel à des orientations telle que l'ancrage des apprentissages dans la vie hors de l'école, comme, les "éducations à " et notamment les éducations à l'environnement. Les programmes de sciences actuels, notamment ceux de sciences de la vie et de la Terre au secondaire inférieur en France (MEN, 2015) véhiculent un discours sur les finalités qui rejoint les trois dimensions exposées ci-dessus. Ce discours reconnait que les $\mathrm{S}$ et $\mathrm{T}$ constituent une des manifestations de la pensée humaine qu'il est important de développer chez les élèves, que ce champ vise à décrire et à expliquer le monde en faisant appel notamment à la conceptualisation et aux démarches d'investigation scientifique (dimension disciplinaire), que son apprentissage doit se faire en lien avec les problématiques sociales et avec la vie à l'extérieur de l'école (dimension sociale).

12 Cependant, l'articulation des savoirs provenant des trois dimensions citées précédemment ne va pas de soi. Les travaux de recherche actuels montrent notamment que l'articulation des DIS avec les savoirs conceptuels semble limitée (Perron, Hasni, Boilevin, 2020 ; Perron, 2018; Marlot et Morge, 2016). Par ailleurs, d'autres études mettent en évidence que les pratiques effectives concernant les «éducations à » et notamment les "éducations au développement durable» correspondent soit à une approche utilitariste et comportementaliste, soit à une continuité " environnementaliste" (Barthes, Lange, et Tutiaux-Guillon, 2017). En effet, Lange relate qu'au primaire, l'approche privilégiée par les enseignants prend souvent la forme "d'une éducation aux "éco-gestes" et aux "bonnes pratiques" moralisatrices sans perspective critique. Ainsi, le tri des déchets devient une activité répandue, parfois complétée par des études faunistiques et floristiques comme approche de la biodiversité » (Barthes, Lange, et Tutiaux-Guillon, 2017, p. 86). Au secondaire, les thématiques du développement durable (production alimentaire, etc.) sont le plus souvent des prétextes pour motiver les activités de classes coutumières.

13 Par ailleurs, dans une société où il semble indispensable de renforcer les liens entre les sciences et la cité, des projets de «sciences participatives» se développent et notamment à destination des élèves.

\section{Les « sciences participatives »}

De nombreux projets de «sciences participatives » ont vu le jour ces dernières années et notamment des projets portant sur des problématiques environnementales. Certains de ces projets sont à destination des élèves.

\section{Une variété de projets}

La participation de non scientifique professionnel ${ }^{2}$ à la production de nouveaux savoirs n'est pas nouvelle. Cependant, les «sciences participatives» ont connu depuis une quinzaine d'années une évolution importante en France et dans le monde (Houllier et Merilhou-Goudard, 2016). À titre d'exemple, les chercheurs du Museum national d'histoire naturelle considèrent que les observations des amateurs sont indispensables 
pour suivre l'évolution de la biodiversité (Couvet et coll., 2011) et mettent en avant le nombre d'heures et la diversité des sites d'observation qui permettent d'obtenir des quantités de données essentielles pour analyser, notamment, les liens entre changement climatique et migrations d'oiseaux ou de papillons (Devictor et coll., 2012). Il existe une forte diversité de projets de « sciences participatives ». En effet, ces projets peuvent porter sur une pluralité de disciplines, de sujets, d'individus impliqués, de méthodes et de finalités. Cette diversité engendre des définitions et des typologies multiples. À titre d'exemple, nous pouvons citer l'utilisation par les chercheurs des termes de «citizen science», "collaborative science», "public engagement", "crowdsourcing", etc. Houllier et Merilhou-Goudard définissent les sciences participatives «comme les formes de production de connaissances scientifiques auxquelles des acteurs non-scientifiques-professionnels, qu'il s'agisse d'individus ou de groupes, participent de façon active et délibérée » (Houllier et Merilhou-Goudard, 2016, p. 12). Différents niveaux de participation des citoyens sont observés. À ce titre, certains auteurs ont réalisé des typologies pour tenter de caractériser ces projets de "sciences participatives " selon divers critères et tout particulièrement la participation des différents acteurs (Houllier et Merilhou-Goudard, 2016; Haklay, 2015; Marjolaine Giroux, 2011). Par exemple, la typologie proposée par Haklay (2015) se compose de quatre niveaux: 1) le crowdsourcing (les citoyens collectent des données); 2) l'intelligence distribuée (les citoyens contribuent à l'analyse des données) ; 3) la science participative (les citoyens participent à la définition du problème et à la collecte de données) ; 4) la collaboration complète (les citoyens sont investis dans l'ensemble des phases de la recherche). Au sein des projets de "sciences participatives" français, l'implication des citoyens se limite majoritairement aux étapes qui demandent une instrumentation (acquisition de données).

Il semble que les projets de "sciences participatives" peuvent apporter à la fois aux sciences et à la société (Wals, Brody, Dillon et Stevenson, 2014). En effet, ils produisent deux types de bénéfices 1) des bénéfices en termes de production de nouveaux savoirs; 2) des bénéfices d'ordre social ou sociétal (Sauermann et Franzoni, 2015). En effet pour les scientifiques, ces projets permettent notamment de mobiliser un grand nombre d'individus pour collecter des données à des échelles à la fois de temps et d'espace plus grandes. Pour les volontaires des projets de «sciences participatives", leur participation favoriserait à titre d'exemple, l'amélioration des rapports sciences-société et l'appropriation des démarches scientifiques (Houllier et Merilhou-Goudard, 2016; Trumbull, Bonney, Bascom et Cabral, 2000). Cependant, ces bénéfices peuvent être contrebalancés notamment par des difficultés à mobiliser les différents acteurs impliqués dans le projet et par des données recueillies par le grand public parfois difficiles à analyser (moindre qualité des données).

Certains projets de «sciences participatives » font intervenir un public particulier : des enseignants et leurs élèves.

\section{Une particularité : « les sciences participatives » à destination des élèves}

Comme pour les projets de «sciences participatives » qui ciblent davantage des adultes ou des individus hors du milieu scolaire, il existe une grande variété de projets destinés spécifiquement aux enseignants et à leurs élèves (tableau 1). 
Tableau 1 : quelques projets de « sciences participatives » à destination des élèves

\begin{tabular}{|l|l|}
\hline \multicolumn{1}{|c|}{ Pays } & \multicolumn{1}{c|}{ Projets } \\
\hline \multirow{2}{*}{ France } & Vigie-Nature Ecole \\
\cline { 2 - 2 } & Survivors \\
\cline { 2 - 2 } & « Les gardiens des chênes » \\
\hline Canada & Monarque sans frontière \\
\hline Suède & Mass experiment \\
\hline
\end{tabular}

19 Les résultats des recherches portant sur les projets de « sciences participatives » en lien avec le milieu scolaire sont mitigés à la fois pour les scientifiques qui proposent ces projets et pour les élèves au regard de l'apprentissage des sciences.

Quelques recherches mettent en doute les aspects bénéfiques des projets de « sciences participatives ». Certains auteurs soulignent que l'impact éducatif et social peut être surestimé (Brossard, Lewenstein et Bonney, 2005) tandis que d'autres s'inquiètent de l'exactitude des données collectées par le grand public, en particulier lorsque des écoliers sont impliqués (Burgess et coll., 2016). Il a été proposé que les données collectées par les écoliers peuvent contribuer à la recherche environnementale, à condition que les méthodes de recherche restent simples et requièrent des compétences que les enfants possèdent déjà ou sont en mesure d'acquérir lorsqu'ils sont encadrés par des adultes (Saunders et coll., 2018). De plus, une étude récente a montré que les données collectées par des élèves n'ont pas moins de valeur que les données recueillies par des scientifiques spécialistes d'une autre discipline (Castagneyrol et coll., 2019).

21 Par ailleurs, d'autres études évoquent que la participation des élèves (avec leurs enseignants) à des projets de «sciences participatives " pourrait contribuer à leurs apprentissages scientifiques en termes de savoirs à la fois de la dimension disciplinaire ${ }^{3}$, épistémologique et sociale (Hecker, Haklay, Bowser, Makuch, Vogel et Bonn, 2018 ; Koomen, Rodriguez, Hoffman, Petersen et Oberhauser, 2018). Ces projets seraient tout particulièrement prometteurs quant à leurs potentialités d'apprentissages en termes de DIS par les élèves (Leuenberger, W., Larsen, Leuenberger, J., et Parry, 2019). De plus, ces projets semblent favoriser la motivation et l'intérêt des élèves pour les sciences citoyennes ainsi que le développement d'attitudes positives envers les animaux sauvages (Kelemen-Finan, Scheuch, et Winter, 2018 ; Jenkins, 2011).

Dans ce contexte, il apparait nécessaire de questionner ces projets de «sciences participatives " notamment dans le champ de la didactique des sciences. Quelles sont les visées éducatives de ces projets (pour les scientifiques et pour les enseignants)? Quelles sont les pratiques des enseignants? Quels sont les apprentissages réalisés par les élèves?

23 Nous avons donc réalisé une première étude afin d'éclairer les pratiques des enseignants lorsqu'ils participent avec leurs élèves à un projet de «sciences participatives ». Notre étude porte sur un projet de «sciences participatives » développé et déployé par des chercheurs de l'INRAE, le projet «Les gardiens des chênes ».

24 Afin d'apporter des éléments de réponse à cet objectif général de recherche, dans la section qui suit, nous explicitons notre cadre de référence. 


\section{Cadre de référence et objectifs spécifiques de recherche}

Notre recherche s'appuie sur un cadre de référence qui articule deux construits : les savoirs en sciences et les « sciences participatives ».

Nous nous appuyons sur les travaux de Hasni et Bousadra (2017), évoqués précédemment, qui dégagent trois principales dimensions qu'il est possible, voire nécessaire, de considérer pour une éducation scientifique et technologique pour tous les élèves allant du primaire au secondaire. Ces dimensions sont considérées ici comme un modèle, une manière simplifiée, de catégoriser les apprentissages scientifiques scolaires: 1/la dimension disciplinaire qui regroupe les savoirs conceptuels, les habiletés, les attitudes, et démarches scientifiques; 2/la dimension sociale qui se compose des savoirs en lien avec les problématiques de la vie hors de l'école (responsabilités individuelles et collectives vis-à-vis des questions de société) et 3/ la dimension épistémologique qui est constituée des savoirs sur les savoirs scientifiques.

De plus, nous pensons comme d'autres auteurs que la construction des savoirs conceptuels peut être vue comme un processus circulaire (figure 2). Hasni et Bousadra (2018) insistent sur l'importance de savoirs préalablement acquis par les élèves pour construire un problème et la nécessité d'utiliser une démarche de recherche pour résoudre un problème. La construction de problèmes est indispensable à l'appropriation des savoirs scientifiques et ces derniers sont nécessaires à la construction des problèmes. En effet, les élèves sont appelés à s'interroger sur des phénomènes scientifiques qui les entourent et amenés à formuler des problèmes d'ordre scientifique sur la base de leurs savoirs conceptuels préalables. Pour trouver des réponses à leurs questions ou des solutions à leurs problèmes, ils planifient et mettent en œuvre, notamment, des démarches d'investigation scientifiques (DIS) appropriées aux questions ou aux problèmes considérés. La formulation des problèmes et la mise en œuvre des DIS nécessitent la mobilisation d'habiletés de recherche et d'attitudes. Au terme de ces démarches, les élèves sont conviés à formuler des énoncés scientifiques et à reconstruire des savoirs scientifiques (savoirs conceptuels visés) afin de mieux comprendre les phénomènes scientifiques en jeu. Les nouveaux savoirs scientifiques ainsi reconstruits peuvent servir à leur tour à la formulation de nouveaux problèmes d'ordre scientifique. Ce processus circulaire de reconstruction des savoirs scientifiques met l'accent sur la relation entre les DIS et la conceptualisation. 


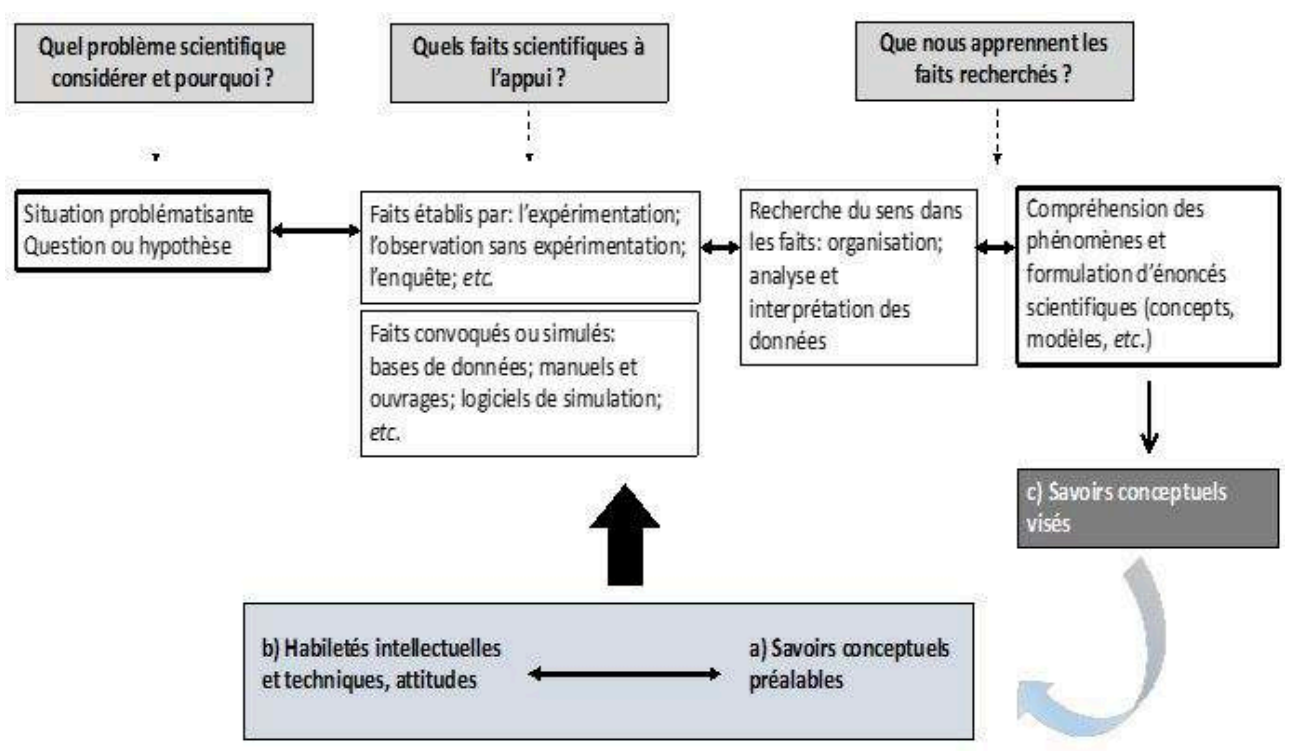

Figure 2 : La reconstruction des savoirs conceptuels à l'école : un processus circulaire (Perron, Hasni et Boilevin, 2020 ; Hasni et Bousadra, 2017)

Enfin, comme Houllier et Merilhou-Goudard nous pensons que les sciences participatives sont «des formes de production de connaissances scientifiques auxquelles des acteurs non-scientifiques-professionnels qu'il s'agisse d'individus ou de groupes participent de façon active et délibérée » (Houllier et Merilhou-Goudard, 2016, p. 6). Cependant, nous considérons que bien que les enseignants soient entièrement volontaires, leurs élèves étant dans un cadre scolaire sont eux plus ou moins contraints de participer. Par conséquent, les élèves ne contribuent pas aux projets de sciences participatives de façon délibérée. Nous formulons donc la définition suivante des sciences participatives quand celles-ci font intervenir des élèves: les sciences participatives, dans un cadre scolaire, sont des formes de production de connaissances scientifiques auxquelles des élèves accompagnés par leurs enseignants participent de façon active. Nous rejoignons également d'autres auteurs qui ont montré l'existence de projets de sciences participatives différents, notamment suivant le degré de participation et les moments de la démarche scientifique pris en charge par les citoyens (Giroux, 2011).

29 En nous appuyant sur notre cadre de référence, notre étude cherche à d'identifier tout particulièrement: 1/les objectifs d'apprentissage pour les élèves; 2/les limites du projet ainsi que les difficultés rencontrées par les enseignants ; $3 /$ les interactions entre les différents participants au projet (enseignant, élèves et chercheurs).

\section{Méthode de recherche}

Il s'agit d'une première étude, de type exploratoire et de nature descriptive. Elle s'appuie sur les pratiques déclarées des enseignants interrogés.

31 La population de référence correspond à l'ensemble des enseignants exerçant en France, s'étant inscrite pour participer au projet de sciences participatives durant l'année scolaire 2018-2019, mis en place par des chercheurs de l'INRAE $(\mathrm{N}=18)$. L'échantillon constitué a posteriori est composé des enseignants volontaires pour 
participer à notre étude $(\mathrm{N}=12)$. Ils sont sollicités par courriel pour répondre à un questionnaire en ligne construit avec le logiciel LimeSurvey. Ce questionnaire est constitué de 56 questions dont 25 questions ouvertes réparties au sein de trois parties : 1/Vos caractéristiques personnelles ; $2 /$ Le projet «Les gardiens des chênes » et 3/La mise en œuvre du projet «Les gardiens des chênes » en classe. Avant d'être diffusé, le questionnaire a été validé par l'ensemble des chercheurs ayant participé à cette étude. Il a également été testé auprès d'un groupe d'enseignants (4) pour s'assurer notamment de la compréhension des questions. Nous cherchons donc ici à travers les données obtenues par ce questionnaire à appréhender (de façon macroscopique) les pratiques déclarées des enseignants.

Les données sont analysées suivant une double approche. Les données provenant des réponses aux questions fermées sont analysées avec le logiciel SPSS avec lequel sont réalisées des statistiques descriptives univariées. Celles issues des réponses aux questions ouvertes sont analysées en utilisant une approche thématique par catégorisation (Bardin, 2007).

\section{L'exemple du projet " Les gardiens des chênes »: les premiers résultats}

\section{Description et caractérisation du projet « Les gardiens des chênes » : une analyse a priori}

\section{Le contexte scientifique du projet}

Six pour cent, c'est la surface foliaire moyenne consommée par les insectes herbivores sur les arbres à l'échelle du globe. Cela paraît peu, mais c'est suffisant pour ralentir la croissance des arbres. Alors que la demande en bois ne cesse d'augmenter, que ce soit pour l'ameublement, le chauffage, la construction ou la production de carton et de papier, il est important de comprendre quels sont les facteurs qui régulent les dégâts causés aux arbres par les insectes herbivores. Les arbres ne sont pas sans défense vis-àvis des insectes herbivores. Leurs feuilles ont des propriétés physiques et chimiques qui limitent la consommation des insectes. Par exemple, les feuilles renferment une grande quantité de composés chimiques qui peuvent réduire la digestibilité ou être toxiques pour les herbivores. Les arbres bénéficient également de défenses indirectes, apportées par les prédateurs et les parasitoïdes. En consommant les herbivores, prédateurs et parasitoïdes limitent la pression d'herbivores sur les arbres. Le climat a des effets directs sur les arbres et leurs défenses, ainsi que sur les herbivores et leurs ennemis. Par exemple, quelques degrés de plus, et les feuilles se mettent en place plus tôt dans l'année. Quelques degrés de trop en été et la survie des arbres peut être menacée. Côté insectes, les hivers plus doux pourraient limiter la mortalité hivernale et par conséquent augmenter le risque de pullulations, ou au contraire empêcher la lever de la diapause hivernale (un processus comparable à l'hibernation). Une augmentation des températures peut également conduire à une accélération du développement des insectes, mais parfois au détriment de leur fécondité. Au final, il est difficile d'anticiper les conséquences du réchauffement climatique sur les dégâts causés par les insectes herbivores. 

d'insectes herbivores sur les arbres tient en partie au fait qu'il est difficile de modifier expérimentalement le climat. Plusieurs études ont bien été réalisées en laboratoire dans des chambres de culture à la température contrôlée, mais elles ont leurs limites. En se focalisant généralement sur une espèce d'arbre (souvent au stade plantule) et une espèce d'herbivore, elles ne peuvent pas rendre compte de la complexité des facteurs qui entrent en jeu dans la régulation des dégâts d'insectes. Une autre approche consiste à réaliser des observations le long de grands gradients géographiques. Il est en effet bien connu que le climat se réchauffe à mesure que l'on se déplace des régions polaires vers l'équateur. Avec cette idée en tête, de nombreux chercheurs ont étudié les dégâts d'insectes, les défenses des arbres, ou encore l'activité des prédateurs, le long de grands gradients latitudinaux. Il est bien établi que le climat influence les interactions arbres insectes, notamment au travers d'un effet sur les défenses de la plante et la qualité des feuilles d'une part, et par le contrôle exercé par les prédateurs d'autre part. Toutefois, l'importance relative de ces facteurs est encore mal comprise, en grande partie parce que les différents facteurs susceptibles d'affecter les dégâts d'insectes herbivores sont généralement étudiés séparément alors qu'ils agissent de concert (Lövei et Ferrante, 2017 ; Roslin et coll. 2017 ; Valdés-Correcher et coll. 2019).

L'étude "Les gardiens des chênes » cherche à déterminer comment le climat influence les dégâts causés par les insectes herbivores sur le chêne pédonculé Quercus robur. Le projet a été mené par 27 scientifiques professionnels dans 13 pays, aidés des élèves de 35 établissements scolaires dans 7 pays. Scientifiques professionnels et élèves ont mesuré la même année et sur les mêmes arbres les dégâts d'insectes défoliateurs, les défenses chimiques et l'activité des prédateurs sur 153 chênes pédonculés adultes le long d'un double gradient géographique allant du Portugal à la Finlande et de l'Angleterre à la Biélorussie.

\section{La méthodologie du projet}

Depuis une dizaine d'années, une méthode simple, efficace, ludique et peu coûteuse s'est imposée dans la communauté scientifique pour estimer l'activité des prédateurs, des herbivores : la mise en place, sur les végétaux, de leurres en pâte à modeler imitant la forme et la taille de vraies chenilles (Roslin et coll. 2017 ; Valdés-Correcher et coll. 2019). Les prédateurs tombent dans le piège: ils attaquent les leurres comme s'il s'agissait de vraies proies et laissent au passage des traces de bec, de dents ou de mandibules, qu'il suffit ensuite de dénombrer. En s'appuyant sur cette méthode, les scientifiques de l'INRAE ont conçu et mis à la disposition des enseignants un protocole que les élèves doivent respecter scrupuleusement ${ }^{4}$. Les classes participant au projet "Les gardiens des chênes " transmettent deux types de données aux écologues : $1 /$ des feuilles de chênes et $2 /$ les leurres après avoir été séjournés 15 jours sur les branches de l'arbre. 


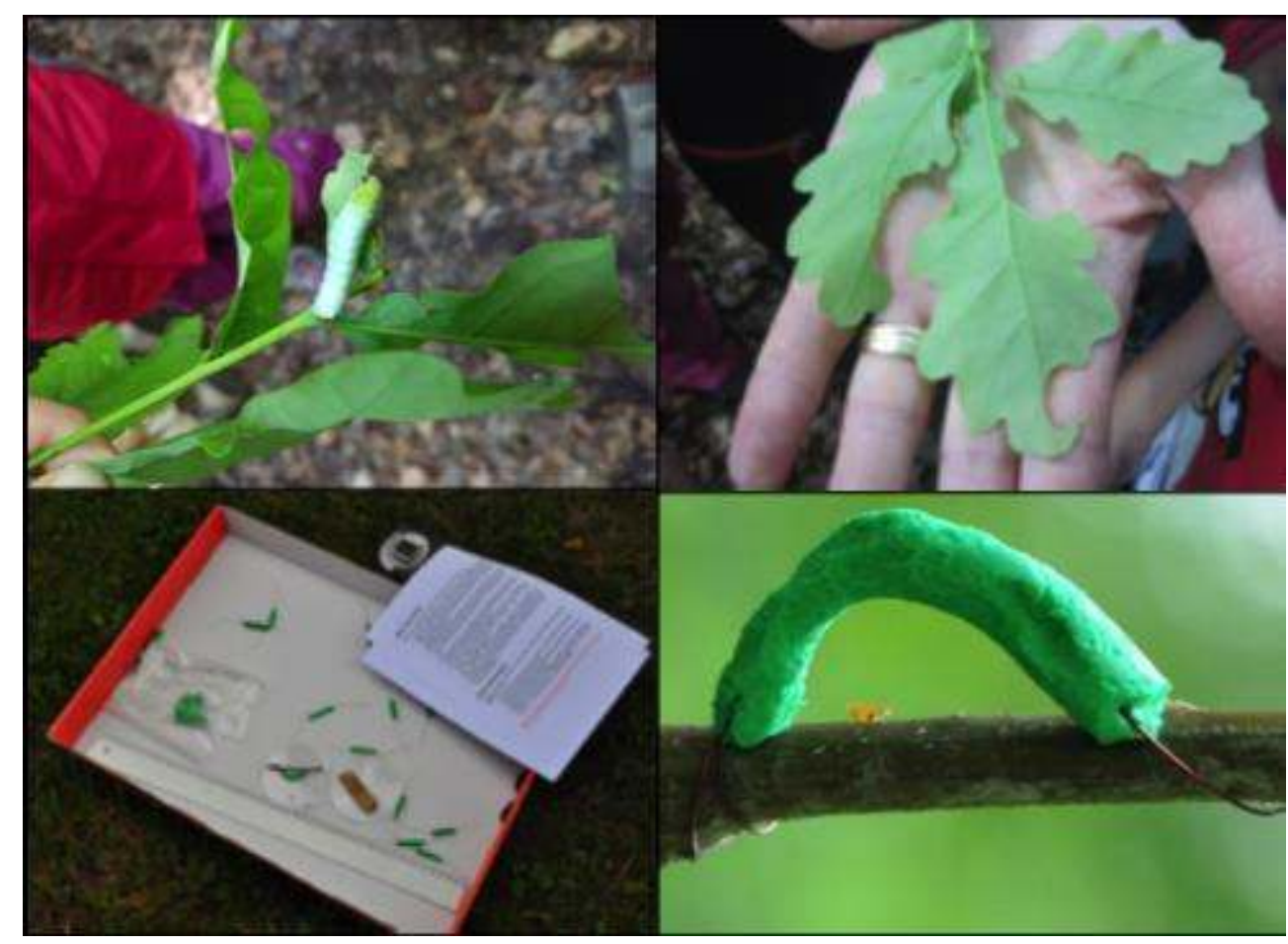

Figure 3 : Photographies illustrant le projet « Les gardiens des chênes »

\section{Un projet de sciences citoyennes}

37 Ce projet destiné aux élèves s'intéresse aux interactions entre sciences et société, c'està-dire comme explicité précédemment, aux formes de production de connaissances scientifiques auxquelles des élèves encadrés par leurs enseignants, participent de façon active. Plus particulièrement, il s'inscrit dans les sciences citoyennes (Giroux, 2011). En effet, le projet «Les gardiens des chênes » déployé sur le territoire français par des chercheurs de l'INRAE est un projet de crowdsourcing faisant intervenir des élèves au niveau de la captation des données. Il s'agit également d'un projet de type « exploratoire » extensif dont l'objectif est de produire des connaissances en lien avec le domaine de l'écologie (Couvet et Teyssèdre, 2013).

\section{Un ancrage éducatif}

Les concepteurs du projet «Les gardiens des chênes » ont clairement en plus de leurs objectifs scientifiques, le souhait de participer à l'éducation scientifique des jeunes citoyens. Ils souhaitent tout particulièrement sensibiliser les élèves aux conséquences du réchauffement climatique sur la biodiversité (en lien avec une éducation à l'environnement alliant la dimension disciplinaire et sociale) et aux méthodes scientifiques (en lien avec la dimension épistémologique). En effet, des ressources à destination des enseignants et des élèves ont été conçues par les scientifiques et les collaborateurs de "Les gardiens des chênes ». Ces ressources sont en accès libre et téléchargeables sur un site internet dédié au projet ${ }^{5}$. Nous pouvons notamment citer une bande dessinée "les gardiens du chêne " qui met en scène la participation de collégiens au projet. Des éléments de vulgarisation scientifique sont également mis à disposition des enseignants afin qu'ils puissent mieux appréhender les enjeux 
scientifiques du projet et les aborder avec leurs élèves. Ces éléments de savoir portent par exemple sur "la cascade trophique » ou sur "les défenses des plantes». Nous précisons que ces ressources ont été pensées et construites par les scientifiques et les collaborateurs du projet.

\section{Le profil des enseignants participants}

Notre échantillon est uniquement composé de femmes ${ }^{6}$. Ce sont des enseignantes expérimentées (11 enseignantes sur 12 exercent depuis plus de 10 ans). Deux d'entre elles sont également formatrices. 3 de ces enseignantes exercent à l'école primaire et 9 enseignent au niveau secondaire ( 4 dans le secondaire supérieur et 5 dans le secondaire inférieur). Parmi ces dernières, 3 ont obtenu le concours de l'agrégation et 4 le concours du Capes (Certificat d'Aptitude au Professorat de l'Enseignement du Second degré).

Par ailleurs, 10 enseignantes sur 12 de notre échantillon ont suivi une formation initiale scientifique. Parmi ces dernières, 9 justifient d'une formation à dominante biologie et/ ou géologie. Seules 2 enseignantes (sur 12) possèdent un diplôme non scientifique (figure 4).

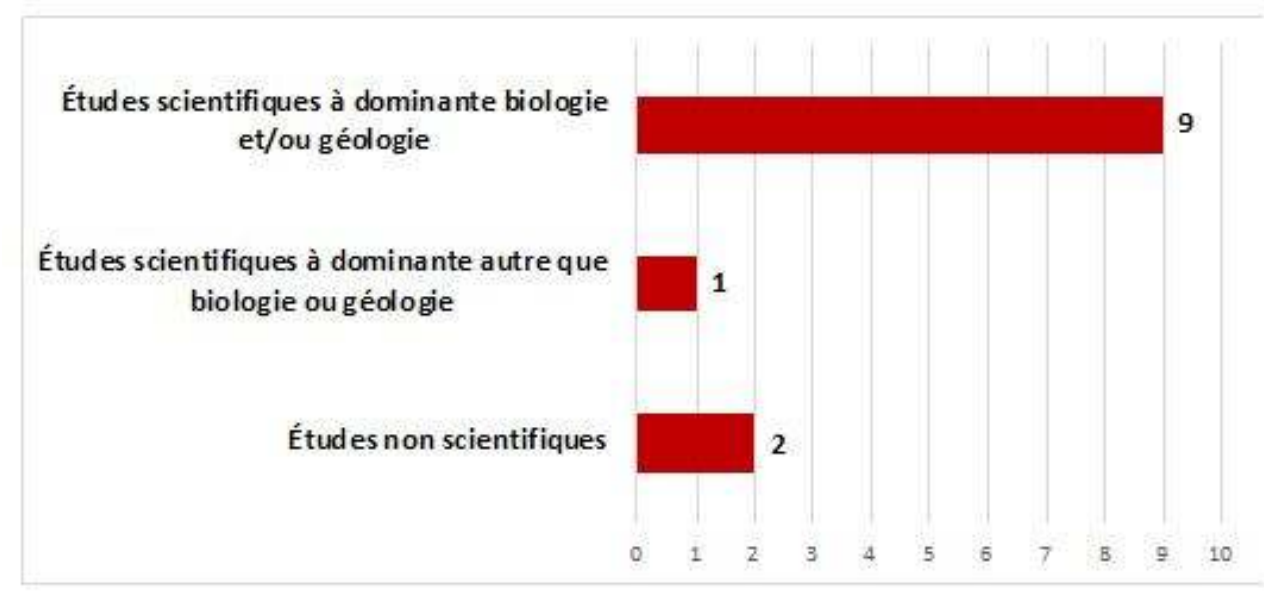

Figure 4 : répartition des enseignantes suivant la formation initiale suivie

En outre, la moitié des enseignantes interrogées ont déclaré avoir déjà participé à un projet de «sciences participatives " (6 sur 12). 5 enseignantes ont pris part à un projet de "sciences participatives" à titre personnel (exemple: Vigie nature papillons ou recensement des oiseaux LPO) et une enseignante s'est impliquée avec ses élèves dans un projet (Sauvage de ma rue Tela Botanica) (figure 5). Cependant, il s'agit uniquement des enseignantes exerçant dans le secondaire. 


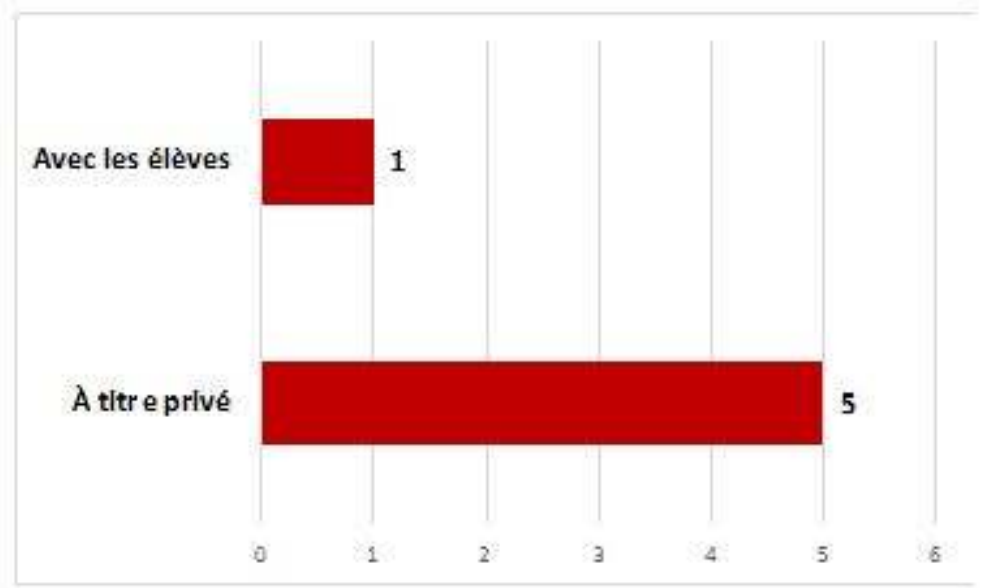

Figure 5 : répartition des enseignantes suivant leur(s) participation(s) antérieure(s) à un projet de sciences participatives

Finalement, ce sont des enseignantes plutôt expérimentées avec un cursus initial tourné vers les sciences qui ont tendance à participer aux projets de «sciences participatives » proposés par des chercheurs de l'INRAE.

Par ailleurs, il est intéressant de noter que ce projet attire des professionnelles sensibles aux «sciences participatives » (la moitié ont déjà participé à un projet), mais pas uniquement. Le profil des enseignants participant au projet «Les gardiens des chênes » est semblable à celui des enseignants inscrits aux différents projets proposés par Vigie nature école (Bosdeveix et coll., 2018).

\section{Objectifs d'apprentissage et savoirs en jeu pour les élèves}

À travers le questionnaire, les enseignantes ont eu l'occasion de s'exprimer concernant l'importance d'objectifs proposés en lien avec le projet "Les gardiens des chênes » (figure 6). Il est possible d'observer que l'objectif «travailler une démarche scientifique » semble le plus important pour les enseignantes interrogées. En effet, elles déclarent à l'unanimité que c'est un objectif très important ou important. En lien avec les DIS, 9 sur 10 considèrent que « mettre en œuvre un protocole expérimental est très important ou important.

Dans le même sens, "éduquer au développement durable » et "sensibiliser à la biodiversité » paraissent des objectifs importants, voire très importants pour la majorité des enseignantes (8 sur 10). Également, «travailler des attitudes » en lien avec l'éducation à la citoyenneté est important pour 7 sur 10 d'entre elles.

Cependant, malgré une grande importante donnée au DIS, seule une enseignante interrogée déclare comme très important et 4 comme important le fait de «travailler des notions ». De même, aucune enseignante ne considère très important de «travailler des capacités ». 


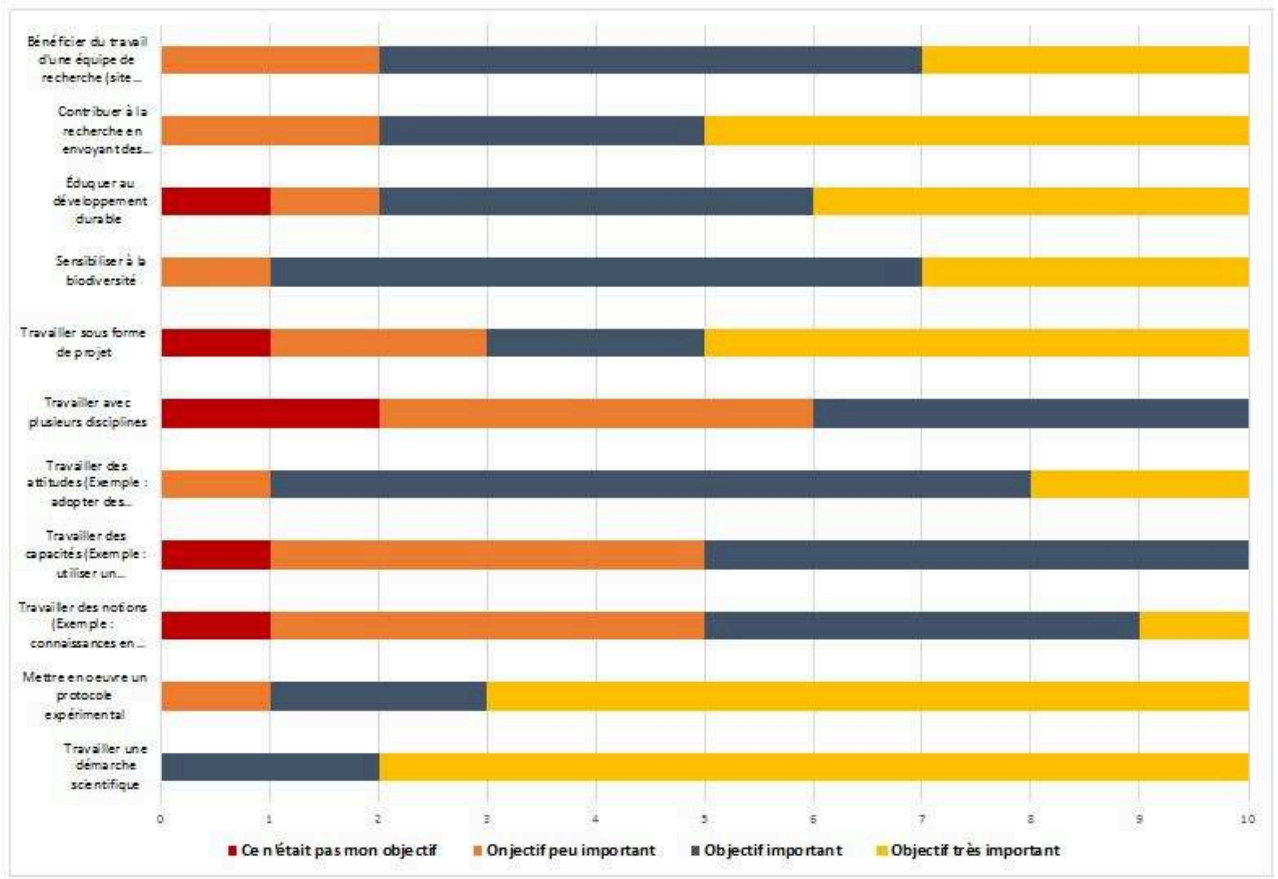

Figure 6 : importance de différents objectifs déclarés les enseignantes interrogées

Lorsqu'il est demandé aux enseignantes ce qu'elles attendent pour leurs élèves du projet de sciences citoyennes de l'INRAE les trois dimensions sont évoquées (tableau 2). 7 évoquent des éléments en relation avec la dimension disciplinaire de l'enseignement des sciences. Cependant en cohérence avec les résultats précédents, il s'agit uniquement ici des DIS ou d'habiletés en lien avec ces démarches comme l'utilisation du protocole. 5 enseignantes déclarent souhaiter que leurs élèves développent des éléments en lien avec la dimension épistémologique. En outre, la dimension sociale que l'on pouvait retrouver dans la figure précédente (figure 5) est peu mise en avant ici par les participantes à notre étude (2 enseignantes). Par ailleurs, nous pouvons noter que certaines enseignantes ont désigné des finalités éducatives d'ordre "pédagogique » $(4$ enseignantes) $)^{7}$. 
Tableau 2 : finalités éducatives envisagées par les enseignantes

\begin{tabular}{|c|c|c|}
\hline Composante & Effectif/10 & Extrait des réponses \\
\hline Disciplinaire & 7 & $\begin{array}{l}\text { «suivre un protocole } » ; \text { « de faire connaitre la } \\
\text { démarche scientifique »; «comprendre la } \\
\text { démarche scientifique » }\end{array}$ \\
\hline Épistémologique & 5 & $\begin{array}{l}\text { «montrer une partie du travail des scientifiques aux } \\
\text { élèves»; «l'importance de la répétitions des } \\
\text { expériences en sciences»; «travailler avec un } \\
\text { chercheur» }\end{array}$ \\
\hline Sociale & 2 & $\begin{array}{l}\text { «une sensibilisation aux enjeux liés au } \\
\text { réchauffement climatique »; "participer à la } \\
\text { préservation de la planète et de la biodiversité » }\end{array}$ \\
\hline Pédagogique & 4 & $\begin{array}{l}\text { «cela m'a permis de développer l'autonomie avec } \\
\text { les élèves »; « le goût de la science »; « susciter } \\
1 \text { 'intérêt pour les sciences» }\end{array}$ \\
\hline
\end{tabular}

Plus précisément, 8 enseignantes de notre échantillon déclarent souhaiter que leurs élèves acquièrent des savoirs lors de la mise en œuvre du projet de sciences citoyennes «Les gardiens des chênes ». Parmi ces enseignantes, 6 évoquent des savoirs en lien avec la dimension disciplinaire (3 des savoirs conceptuels comme « la chaine alimentaire " ou des «notions sur la biodiversité » et 3 les DIS) et 4 notent des savoirs portant le réchauffement climatique ( impacts possibles du réchauffement climatique ») en lien avec la dimension sociale. Nous remarquons également que la majorité des enseignantes souhaitant que leurs élèves acquièrent des savoirs, nomme un savoir en particulier. En effet, 6 enseignantes expliquent que leurs élèves doivent acquérir soit des savoirs conceptuels (2), soit des DIS (2) et soit des savoirs liés à citoyenneté (2). Quelques enseignantes (2) semblent désirer que leurs élèves acquièrent différents savoirs : des savoirs conceptuels et des savoirs de la dimension sociale (1) et des DIS et des savoirs de la dimension sociale (1).

En outre, seules 4 des enseignantes interrogées expliquent désirer que leurs élèves mobilisent des savoirs préalablement acquis lors de la mise en œuvre du projet. Seuls les savoirs composant la dimension disciplinaire sont évoqués. La moitié parlent de DIS et de savoirs conceptuels comme « la chaine alimentaire » ou " les réseaux trophiques " et une enseignante nomme des habiletés intellectuelles telle que " la curiosité ».

Concernant, les composantes des DIS, seules 4 des enseignantes interrogées et uniquement des enseignantes du secondaire, relatent qu'un problème scientifique a été formulé pendant les séances consacrées au projet. De plus, les «problèmes » notés par ces derniers sont davantage des questions («Pour les élèves, tout était problème et recherche de compréhension »).

51 Finalement, les savoirs composant la dimension disciplinaire, avec les DIS et les habiletés en lien avec ces démarches comme l'utilisation d'un protocole expérimental, semblent occuper une place centrale pour les enseignantes lors de la mise en œuvre par leurs élèves du projet de sciences citoyennes de l'INRAE. Ce même résultat a déjà été mis en évidence par Bosdeveix et coll. (2018). Cependant, les savoirs conceptuels bien qu'évoqués par les enseignantes paraissent de moindre importance, voire presque inexistants lorsqu'il s'agit de savoirs conceptuels préalablement acquis par les élèves. 
52 Les savoirs en lien avec la dimension sociale concernant le réchauffement climatique ou la biodiversité semblent également présents lors de la mise en œuvre du projet « Les gardiens des chênes ". Seuls les savoirs sur les savoirs scientifiques (dimension épistémologique) sont absents des savoirs à acquérir et à mobiliser par les élèves (selon les déclarations des enseignantes). En effet, ils font parties des attentes que les enseignantes ont pour leurs élèves à propos du projet, mais ils ne semblent pas concrètement visés.

Il ne parait pas exister de réelle articulation entre les différents savoirs scientifiques issues des trois dimensions lorsque les élèves mettent en œuvre ce projet de sciences citoyennes. De plus, comme cela a déjà été mis en évidence au travers d'une autre étude (Perron, Hasni et Boilevin; Perron, 2018), il existe une dichotomie entre DIS et les savoirs conceptuels. Les DIS sont étudiées pour elles-mêmes.

\section{Difficultés rencontrées par les enseignantes}

La plupart des enseignantes interrogées estiment avoir rarement rencontré de difficultés lors de la mise en œuvre du projet «Les gardiens des chênes ». Seules deux enseignantes du primaire relatent avoir souvent été confrontées à des difficultés.

Les difficultés évoquées par les enseignantes sont de nature diverse (tableau 3). Il est possible de noter que certains enseignants (2) ont rencontré des difficultés concernant l'interprétation des prédations (description des résultats). L'adéquation entre le temps scolaire et la temporalité imposée par la rigueur du protocole expérimental du projet a paru compliqué pour presque un tiers des enseignantes. Pour surmonter ces difficultés, les enseignantes interrogées évoquent notamment souhaiter davantage de collaboration réelle entre les chercheurs et les élèves (3) et expriment qu'il est nécessaire de bien préparer la séquence d'enseignement en amont.

Tableau 3 : les difficultés rencontrées par les enseignantes et ce qui leur a manqué pour les surmonter

\begin{tabular}{|l|c|l|c|}
\hline \multicolumn{1}{|c|}{$\begin{array}{c}\text { Difficultés } \\
\text { évoquées }\end{array}$} & Fréquence/10 & $\begin{array}{c}\text { Manques pour surmonter } \\
\text { ces difficultés }\end{array}$ & Fréquences/10 \\
\hline Temporalité & 3 & $\begin{array}{l}\text { Collaboration avec entre } \\
\text { élèves chercheurs }\end{array}$ & 3 \\
\hline Météo & 2 & Planification enseignant & 3 \\
\hline $\begin{array}{l}\text { Accessibilité au site } \\
\text { d'étude (arbres) }\end{array}$ & 2 & Plus de temps & 2 \\
\hline $\begin{array}{l}\text { Interprétation des } \\
\text { prédations }\end{array}$ & 2 & $\begin{array}{l}\text { Documents fournis par les } \\
\text { chercheurs }\end{array}$ & 2 \\
\hline $\begin{array}{l}\text { Action limitée des } \\
\text { élèves }\end{array}$ & 1 & & \\
\hline
\end{tabular}

57 Finalement peu d'enseignantes déclarent avoir rencontré des difficultés lors de la mise en œuvre du projet avec leurs élèves. Néanmoins, elles semblent souhaiter davantage de relations entre les scientifiques et leurs élèves. Peut-être que cette collaboration 
permettrait une articulation entre les différentes dimensions disciplinaire, épistémologique et sociale.

\section{Interactions entre les différents acteurs (chercheurs, enseignantes, élèves)}

Les enseignantes de notre échantillon semblent avoir été peu en contact avec les chercheurs de l'INRAE. En effet, 3 d'entre elles déclarent avoir été parfois en contact avec les chercheurs et 5 avoir été rarement ou très rarement en contact (figure 7). De plus, l'ensemble des enseignantes exprime ne pas souhaiter avoir davantage de contact. Une certaine flexibilité de la part des chercheurs qui ont su s'adapter aux demandes des enseignantes semble avoir convenu à ces dernières.

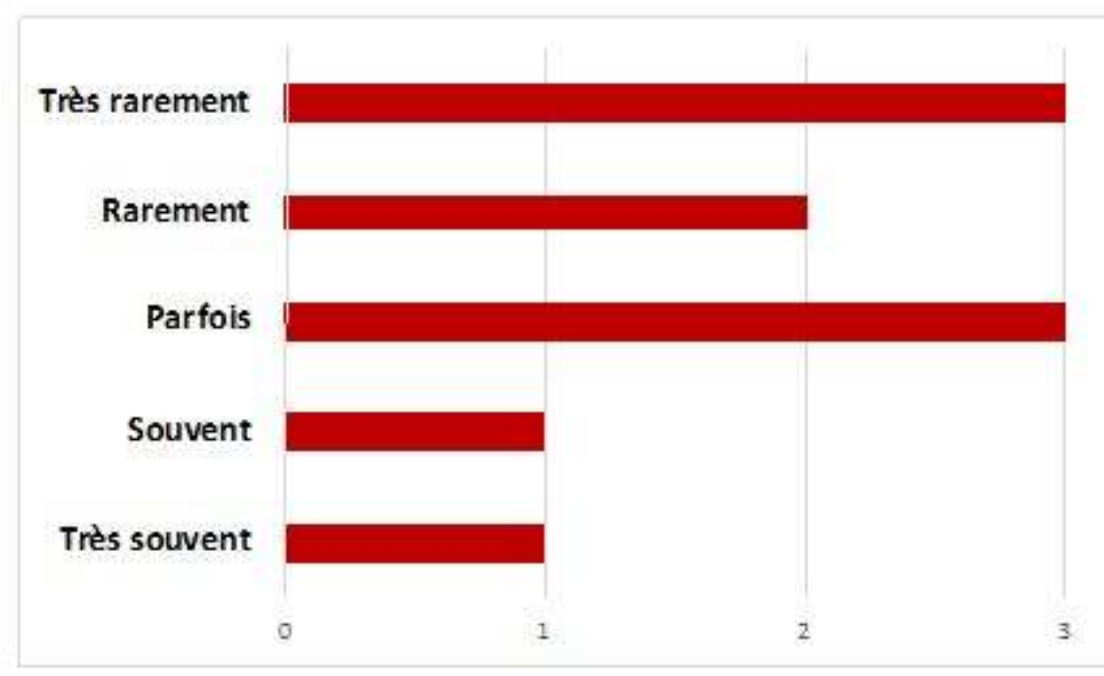

Figure 7 : fréquence des contacts entre les enseignantes et les chercheurs de l'INRAE

La quasi-totalité des contacts se sont déroulés avant la mise en œuvre du projet. Les enseignants ont souhaité davantage d'explicitation du projet et du protocole. Par ailleurs, l'ensemble de ces enseignantes déclare avoir communiqué par courrier électronique avec les chercheurs. Seule une enseignante relate avoir également pris contact par téléphone et avoir rencontré réellement les scientifiques. Cette rencontre a eu lieu en classe avec les élèves pour présenter le projet.

Finalement, peu de contacts ont eu lieu entre les différents acteurs (enseignantes, chercheurs et élèves) et quasi exclusivement entre les enseignantes et les chercheurs de façon virtuelle (figure 8). 


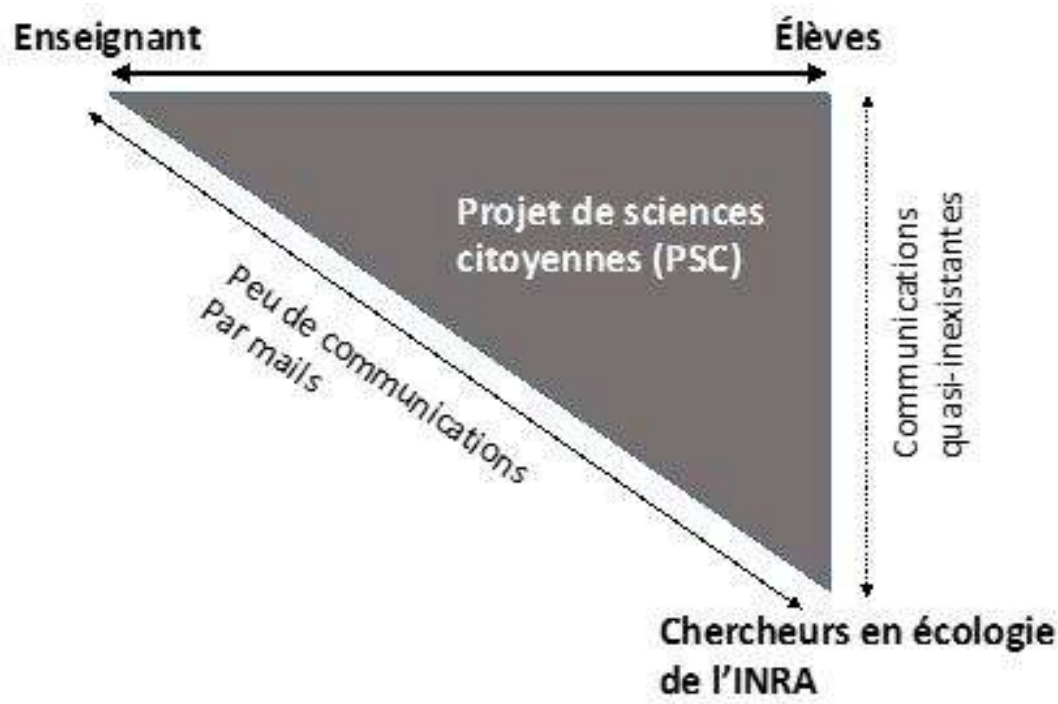

Figure 8 : représentation des relations entre les différents participants au projet

Lorsque nous demandons aux enseignantes « Pouvez-vous décrire quelle collaboration vous auriez souhaitée avec les chercheurs de l'INRAE ? ", 4 enseignantes déclarent ne rien souhaiter de plus (la collaboration instaurée semble avoir convenu), 3 auraient aimé que les chercheurs échangent avec leurs élèves et 3 désireraient obtenir les résultats de l'étude à laquelle elles ont participées (tableau 4).

Tableau 4 : collaboration souhaitée par les enseignantes

\begin{tabular}{|l|c|l|}
\hline \multicolumn{1}{|c|}{$\begin{array}{c}\text { Collaboration } \\
\text { souhaitée }\end{array}$} & Effectif/10 & \multicolumn{1}{|c|}{ Extrait des réponses } \\
\hline Obtenir les résultats & 3 & $\begin{array}{l}\text { «nous attendons les conclusions de } \\
\text { l'enquête» } \\
\text { «J'aimerais un retour des résultats ... ou de } \\
\text { la conclusion.... » }\end{array}$ \\
\hline $\begin{array}{l}\text { Des échanges entre } \\
\text { élèves et chercheurs }\end{array}$ & 3 & $\begin{array}{l}\text { « une visioconférence pour expliquer aux } \\
\text { élèves l'expérience : pourquoi-comment } \\
\text { « une intervention d'un chercheur auprès } \\
\text { des élèves » }\end{array}$ \\
\hline Rien de plus & 4 & \\
\hline
\end{tabular}

Nous pensons donc qu'une collaboration plus importante entre les différents acteurs (scientifiques, élèves et enseignants) serait souhaitable pour que les enseignants et les élèves puissent appréhender le projet dans sa complexité au travers des trois dimensions éducatives. Par ailleurs, cette collaboration permettrait aux scientifiques une meilleure compréhension du milieu scolaire leur donnant l'occasion de mieux cerner les attentes et les besoins des enseignants et de leurs élèves. 


\section{Conclusion : une collaboration entre scientifiques, enseignants et élèves nécessaire pour appréhender la complexité d'un projet de sciences citoyennes?}

63 Nous avons donc mené une étude de type exploratoire qui s'intéresse à un projet de sciences citoyennes développé par des scientifiques de l'INRAE, le projet «Les gardiens des chênes ». Cette étude, qui s'appuie sur des pratiques déclarées de 12 enseignants, avait pour objectif d'identifier les visées éducatives des enseignants. Les difficultés rencontrées par les enseignants ainsi que les interactions entre les différents acteurs (enseignants, les scientifiques et élèves) ont également été mises en évidence.

Les résultats montrent que les visées éducatives principales des enseignants de notre échantillon sont directement en lien avec les DIS. Ces résultats confirment les travaux réalisés par Bosdeveix et ses collègues (2018). La dimension sociale est néanmoins présente, mais la dimension épistémologique est quasiment absente. Il ne parait pas exister de réelle articulation entre les différentes dimensions disciplinaire, épistémologique et sociale. De plus, comme d'autres recherches l'ont déjà mise en évidence la construction de savoirs conceptuels ne fait pas partie des objectifs d'apprentissage (Perron, 2018). Les DIS sont étudiées pour elles-mêmes. Il n'y a pas non plus d'articulation eu sein de la dimension disciplinaire.

Par ailleurs, les scientifiques ont eu très peu de contact avec les enseignantes, voir aucun avec leurs élèves. Pourtant, certaines études ont montré que des échanges directs entre des élèves et des scientifiques au sein de leurs laboratoires permettent à ces élèves une meilleure compréhension de la nature des sciences (Woods-Townsend et coll., 2016 ; Tsybulsky, 2019).

66 La participation des élèves au projet "Les gardiens des chênes " semble se résumer à l'application du protocole construit par les scientifiques et donc uniquement à recueillir des données. Les possibilités d'apprentissages des élèves à travers ce projet paraissent minces en termes de savoirs des dimensions épistémologique et sociale, mais aussi en termes de savoirs conceptuels.

67 Finalement, le projet "Les gardiens des chênes" ne semble pas avoir permis de porosité de la frontière entre les scientifiques et les établissements scolaires. Ces deux mondes sont restés étanches. Le protocole construit par les scientifiques de l'INRAE et mis à la disposition des enseignants sur la page Web du projet ne constitue pas un objet frontière. Ces constats ne paraissent pas donner la possibilité aux élèves d'accéder à la dimension épistémologique des savoirs scientifiques ainsi qu'à leur dimension sociale pourtant présentes dans le projet.

68 En nous appuyant sur ce qui précède, nous pensons néanmoins qu'il est possible d'envisager les projets de sciences citoyennes comme des opportunités d'apprentissages scientifiques complexes pour les élèves en lien avec une éducation à l'environnement. Ces apprentissages se feraient au travers de l'articulation des trois dimensions disciplinaire, épistémologique et sociale. Cependant, nous présumons que cela nécessite une réelle collaboration entre les scientifiques et les enseignants, mais aussi entre les scientifiques et les élèves. À ce titre, des études s'intéressant tout particulièrement aux apprentissages réalisés par les élèves lorsqu'ils participent à des projets de sciences citoyennes sont nécessaires. 


\section{BIBLIOGRAPHIE}

Albe, V. (2008). Pour une éducation aux sciences citoyenne. Une analyse sociale et épistémologique des controverses sur les changements climatiques. Aster, 46.

Bardin, L. (2007). L'analyse de contenu. Paris : Presses Universitaires de France.

Barthes, A., Lange, J-M., et Tutiaux-Guillon, N. (2017). Dictionnaire critique des enjeux et concepts des "éducation à ». Paris : L'harmattan.

Bennett, J., Lubben, F. et Hogarth, S. (2007). Bringing science to life : A synthesis of the research evidence on the effects of context-based and STS approaches to science teaching. Science Education, 91(3), 347-370.

Bosdeveix, R., Crépin-Obert, P., Fortin, C., Leininger-Frézal, C., Regad, L., et Turpin, S. (2018). Étude des pratiques enseignantes déclarées concernant le programme de sciences citoyennes Vigie-Nature École. RDST. Recherches en didactique des sciences et des technologies, 18, 79-102.

Brossard, D., Lewenstein, B., et Bonney, R. (2005). Scientific knowledge and attitude change : The impact of a citizen science project. International Journal of Science Education, 27(9), 1099-1121.

Burgess, H. K., DeBey, L. B., Froehlich, H. E., Schmidt, N., Theobald, E. J., Ettinger, A. K.,... et Parrish, J. K. (2017). The science of citizen science : exploring barriers to use as a primary research tool. Biological Conservation, 208, 113-120.

Castagneyrol, B., Valdés-Correcher, E., Bourdin, A. et coll. (2019). Can school children support ecological research ? Lessons from the 'Oak bodyguard' citizen science project. BioRxiv, 712638.

Couvet, D., Devictor, V., Jiguet, F., et Julliard, R. (2011). Scientific contributions of extensive biodiversity monitoring. Comptes Rendus Biologies, 334(5-6), 370-377.

Couvet, D., et Teyssèdre, A. (2013). Sciences participatives et biodiversité : de l'exploration à la transformation des socio-écosystèmes. Cahiers des Amériques latines, 72-73

DeBoer, G. E. (2000). Scientific literacy : Another look at its historical and contemporary meanings and its relationship to science education reform. Journal of Research in Science Teaching, $37(6), 582-601$.

Devictor, V., Van Swaay, C., Brereton, T., Brotons, L., Chamberlain, D., Heliölä, J. et Reif, J. (2012). Differences in the climatic debts of birds and butterflies at a continental scale. Nature Climate Change, 2(2), 121-124.

Eitzel, M. V., Cappadonna, J. L., Santos-Lang, C., Duerr, R. E., Virapongse, A., West, S. E.,... \& Metcalfe, A. N. (2017). Citizen science terminology matters : exploring key terms. Citizen science : Theory and practice, 2(1).

Eurydice (2006). L'enseignement des sciences dans les établissements scolaires en Europe. États des lieux des politiques et de la recherche. Bruxelles : Commission européenne. Direction Générale de l'Éducation et de la Culture.

Giroux, M. 2011. La participation citoyenne, un outil pour mieux connaitre la biodiversité : portrait nord-américain. Présentation aux Troisièmes Rendez-vous de l'Accord Canada-France, Biodôme de Montréal, 22-26 mars 2011.

Haklay, M. 2015. Citizen Science and Policy : A European Perspective. Washington D.C. : The Woodrow Wilson Center/Commons Lab. 
Hasni, A. et Lebrun, J. (2008). Les valeurs explicites dans les programmes et dans les manuels de sciences et technologies au Québec. Dans, Daniel Favre éd., Les valeurs explicites et implicites dans la formation des enseignants : Entre « toujours plus » et « mieux vivre ensemble » (pp. 101-116). Louvain-laNeuve, Belgique : De Boeck Supérieur.

Hasni, A., et Potvin, P. (2015). Student's Interest in Science and Technology and Its Relationships with Teaching Methods, Family Context and Self-Efficacy. International Journal of Environmental and Science Education, 10(3), 337-366.

Hasni, A et Bousadra, F : (2017). Les finalités éducatives pour les sciences : entre l'idéal des chercheurs et du curriculum et les défis de leur opérationnalisation dans les manuels et dans les pratiques d'enseignement. Dans Y. Lenoir, O. Adiguzel, A. Lenoir, J.C. Libaneo et F. Tupin (dir), Les finalités éducatives scolaires. Une étude critique des approches théoriques, philosophiques et idéologiques. Montréal : Groupédition Éditeurs.

Hasni, A. et Bousadra, F. (2018). Les démarches d'investigation scientifique dans les classes d'enseignants du secondaire au Québec : défis théoriques et pratiques. Dans A. Hasni, F. Bousadra et J. Lebeaume (dir), Les démarches d'investigation scientifique et de conception technologique : Regards croisés sur les curriculums et les pratiques en France et au Québec. Montréal : Groupédition Éditeurs.

Hecker, S., Haklay, M., Bowser, A., Makuch, Z., Vogel J., et Bonn A. (2018). Citizen Science : Innovation in Open Science, Society and Policy (1st ed.).

Jenkins, L. L. (2011). Using citizen science beyond teaching science content : A strategy for making science relevant to students » lives. Cultural Studies of Science Education, 6(2), 501508.

Houllier, F., et Merilhou-Goudard, J. B. (2016). Les sciences participatives en France. États des lieux, bonnes pratiques et recommandations. Rapport de l'INRA (France).

Kelemen-Finan, J., Scheuch, M. et Winter, S. (2018). Contributions from citizen science to science education : An examination of a biodiversity citizen science project with schools in Central Europe. International Journal of Science Education, 40(17), 2078-2098.

Kolstoe, S. D. (2000). Consensus projects : Teaching science for citizenship. International Journal of Science Education, 22(6), 645-664.

Koomen, M. H., Rodriguez, E., Hoffman, A., Petersen, C. et Oberhauser, K. (2018). Authentic science with citizen science and student-driven science fair projects. Science Education, 102(3), 593-644.

Lederman, N.G., Antink, A. et Bartos, S. (2014) Nature of Science, Scientific Inquiry, and SocioScientific Issues Arising from Genetics : A Pathway to Developing a Scientifically Literate Citizenry. Science and Education, 23, 285-302.

Lee, S., et Roth, W.-M. (2003). Science and the « Good Citizen » : Community-Based Scientific Literacy. Science, Technology, and Human Values, 28(3), 403-424.

Leuenberger, W., Larsen, E., Leuenberger, J. et Parry, D. (2019). Predation on Plasticine Model Caterpillars : Engaging High School Students Using Field-Based Experiential Learning Camp ; the Scientific Process. The American Biology Teacher, 81(5), 334-339.

Lövei, G. L., et M. Ferrante. (2017). A review of the sentinel prey method as a way of quantifying invertebrate predation under field conditions. Insect Science, 24, 528-542.

Marlot, C. et Morge, L. (dir.) (2016). L'investigation scientifique et technologique. Comprendre les difficultés de mise en œuvre pour mieux les réduire. Rennes : Presses universitaires de Rennes. 
Ministère de l'Éducation Nationale (MEN) (2015). Programme de sciences et technologie du collège. France : Ministère de l'Éducation Nationale.

Organisation de Coopération et de Développement Économique (OCDE) (2007). Les compétences en sciences, un atout pour réussir, Volume 1 Analyse des résultats. Paris : OCDE.

Perron, S. (2018). Étude de l'articulation des démarches d'investigation scientifique avec les autres savoirs composant la structure disciplinaire : cas d'enseignants de sciences de la vie et de la Terre exerçant en collège français. Thèse de doctorat, Université de Bretagne occidentale-Brest, Université de Sherbrooke (Québec, Canada). Consulté sur https://tel.archives-ouvertes.fr/tel-02083967/ document

Perron, S., Hasni, A. et Boilevin, J.-M. (2020). L'absence de savoir conceptuel lors de démarches d'investigation scientifique mises en œuvre en classe : une crainte devenue réalité ? Recherches en Education, 42.

Roberts, D. A. (2007). Scientific literacy/Science literacy. In S. Abell \& N. G. Lederman (Éd.), Handbook of Research on Science Education (p. 729 780). Laurence Erlbaum Associates.

Roslin, T., B. Hardwick, V. Novotny, W. K.et coll. (2017). Higher predation risk for insect prey at low latitudes and elevations. Science, 356, 742-744.

Sauermann, H. et Franzoni, C. (2015). Crowd science user contribution patterns and their implications. Proceedings of the national academy of sciences, 112(3), 679-684.

Saunders, M. E., Roger, E., Geary, W. L. et coll. (2018). Citizen science in schools : Engaging students in research on urban habitat for pollinators. Austral Ecology, 43(6), 635-642.

Schwab, J. (1964). Structure of the Discipline. Dans G.W. Ford et L. Pugno (dir.), The structure of knowledge and the curriculum (p. 6-30). Chicago : Rand Mcnally et Compagny.

Trumbull, D. J., Bonney, R., Bascom, D. et Cabral, A. (2000). Thinking scientifically during participation in a citizen-science project. Science Education, 84(2), 265-275.

Tsybulsky, D. (2019). Students meet authentic science : The valence and foci of experiences reported by high-school biology students regarding their participation in a science outreach program. International Journal of Science Education, 41(5), 567-585.

Valdes-Correcher, E., van Halder, I., Barbaro, L., Castagneyrol, B. et Hampe, A. (2019). Insect herbivory and avian insectivory in novel native oak forests : Divergent effects of stand size and connectivity. Forest Ecology and Management, 445, 146-153.

Wals, A. E. J., Brody, M., Dillon, J. et Stevenson, R. B. (2014). Convergence Between Science and Environmental Education. Science, 344, 583-584.

Woods-Townsend, K., Christodoulou, A., Rietdijk, W., Byrne, J., Griffiths, J. B. et Grace, M. M. (2016). Meet the Scientist : The Value of Short Interactions Between Scientists and Students. International Journal of Science Education, Part B, 6(1), 89-113.

\section{NOTES}

1. «L'important est que les étudiants apprennent quelque chose qu'ils trouveront intéressant pour qu'à l'avenir, ils continuent d'étudier les sciences de manière à la fois formelle et informelle » (traduction personnelle).

2. Il existe un débat sur la scène scientifique au sujet du ou des termes à employer lorsque l'on parle des personnes (extérieur à la recherche) qui participent à un projet de «sciences 
participatives » (Eitzel, M. V. et coll., 2017). Nous concernant, nous avons fait le choix d'utiliser celui de «non scientifique professionnel». En effet, ce terme nous paraît le plus neutre et le moins excluant.

3. Le terme disciplinaire ici s'emploie de manière générique (au singulier). Cependant, il peut représenter plusieurs disciplines convoquées pour aborder la complexité des problématiques liées à l'environnement.

4. https://www6.INRAE.fr/tree-bodyguards/content/download/3532/34428/version/1/file/ FR_Protocole_2021-02-19.pdf

5. https://www6.inrae.fr/tree-bodyguards/

6. Ce sont des enseignantes inscrites pour participer au projet avec leurs élèves et volontaires pour répondre à notre questionnaire. Il s'agit ici d'un échantillonnage a posteriori.

7. Nous ne développons pas cet élément dans le cadre de cette recherche.

\section{RÉSUMÉS}

Depuis quelques années des projets de sciences citoyennes à destination des élèves, se développent (Castagneyrol et coll., 2019; Bosdeveix et coll., 2018). Ces projets semblent prometteurs quant à leurs potentialités d'apprentissages par les élèves (Leuenberger, W., Larsen, Leuenberger, J., et Parry, 2019). Nous avons donc mené une première étude de type exploratoire qui s'intéresse au projet de sciences citoyennes "Les gardiens des chênes " développé par des scientifiques de l'INRAE. Cette étude, qui s'appuie sur des pratiques déclarées de 12 enseignants, a principalement pour objectif d'identifier les objectifs d'apprentissage des élèves. Les résultats montrent que malgré une importance relative donnée aux dimensions épistémologiques et sociales, les visées éducatives des enseignants de notre échantillon sont presque exclusivement en lien avec les démarches d'investigation scientifique.

In recent years, citizen science projects for students have been developing (Castagneyrol et coll., 2019 ; Bosdeveix et coll., 2018). These projects seem promising in terms of their potential for student learning (Leuenberger, W., Larsen, Leuenberger, J., and Parry, 2019). We therefore carried out an exploratory study which is interested in the citizen science project "Guardians of the Oaks" developed by INRAE scientists. The main objective of this study, which is based on the declared practices of 12 teachers, is to identify the learning objectives of students. The results show that despite a relative importance given to the epistemological and social dimensions, the educational aims of the teachers in our sample are almost exclusively linked to scientific investigation procedures.

\section{INDEX}

Mots-clés : savoirs en sciences, sciences citoyennes, éducation à l'environnement, pratique des enseignants

Keywords : science knowledge, citizen science, environmental education, teaching practices 


\section{AUTEURS}

\section{SÉVERINE PERRON}

Actuellement Maître Assistante (Chercheure séniore) à l'Université de Genève, Séverine Perron participe aux travaux de recherche du Groupe de recherche en didactique comparée (GREDIC). Elle s'intéresse à l'articulation des savoirs scientifiques (dimension disciplinaire, épistémologique et sociale) en classe et aux frontières des écoles. Elle est diplômée d'un doctorat en Didactique des sciences de l'UBO (France) et d'un Ph.D en Éducation de l'Université de Sherbrooke. Courriel : severine.perron[@]unige.ch

\section{PATRICIA MARZIN-JANVIER}

Patricia Marzin-Janvier est Professeure des Universités à l'Université de Bretagne Occidentale, INSPE (Institut National Supérieur du Professorat et de l'Éducation) de Bretagne (France) et codirectrice du Centre de recherche sur l'Éducation, les Apprentissages et la Didactique (CREAD). Ses recherches sont centrées sur les mécanismes de transmission et d'appropriation de savoirs principalement en biologie. Courriel : patricia.marzin-janvier[@]inspe-bretagne.fr

\section{BASTIEN CASTAGNEYROL}

Bastien Castagneyrol est chercheur (écologue) au sein de l'Unité Mixe de Recherche (UMR) Biodiversité Gènes \& Communautés de l'Université de Bordeaux et de l'Institut national de recherche pour l'agriculture, l'alimentation et l'environnement (INRAE, France). Il travaille notamment sur les conséquences du réchauffement climatique sur les dégâts causés par les insectes herbivores sur les arbres. Courriel : bastien.castagneyrol[@]INRAE.fr 